\title{
Pulmonary thromboembolism in children with rheumatic diseases
}

\author{
Larry B Vogler ${ }^{4^{*}}$, Sheila Angeles-Han ${ }^{3}$, Sampath Prahalad ${ }^{2}$, Egla C Rabinovich ${ }^{1}$ \\ From 2011 Pediatric Rheumatology Symposium sponsored by the American College of Rheumatology \\ Miami, FL, USA. 2-5 June 2011
}

\section{Purpose}

To demonstrate the clinical features and predisposing factors of pulmonary thrombotic events in children with rheumatic diseases.

\section{Methods}

Chart review, observational.

\section{Results}

Thrombotic events have been associated with antiphospholipid antibodies in autoimmune diseases, including systemic lupus erythematosus (SLE). However, pulmonary thromboembolism (PTE) from deep vein thromboses (DVT) or in situ pulmonary arterial thrombosis is uncommon in rheumatic diseases, especially in children. The diagnosis and treatment of PTE may be delayed due to a paucity of symptoms or to symptoms attributed to more common manifestations such as pleuritis or pneumonia. We report findings in 6 children with PTE secondary to SLE (4), Systemic Sclerosis (SSc) (1) and Polyarteritis Nodosa (PAN) (1).

\section{Conclusion}

Although antiphospholipid antibodies are common in SLE, pulmonary arterial thrombosis is rare. These 4 cases of SLE represent only $1.7 \%$ of 234 pediatric lupus patients seen at Emory University over 18 years. Pulmonary thromboemboli may mimic pleuritis with effusion or pneumonia. Besides antiphospholipid antibodies, which were present in only 2 of these patients, other

Table 1

\begin{tabular}{|c|c|c|c|c|c|c|}
\hline Pt/Gender & $1 / F$ & $2 / F$ & $3 / \mathrm{M}$ & $4 / F$ & $5 / \mathrm{M}$ & $6 / \mathrm{M}$ \\
\hline \multirow[t]{2}{*}{ Dx } & SLE & SLE & SLE & SLE & SSC & PAN \\
\hline & thrombocytopenia & nephritis (IV) & nephritis (V) & nephritis (IV) & $\mathrm{PAH}$ & CVA \\
\hline Age at Dx (yr) & 12.6 & 14.1 & 9.0 & 12.0 & 12.8 & 0.3 \\
\hline Age at PTE & 15.2 & 14.8 & 16.8 & 12.6 & 16 & $6 / 4$ \\
\hline \multirow[t]{2}{*}{ Symptoms } & leg pain & chest pain & chest pain & chest pain & chest pain & leg pain \\
\hline & & dyspnea & dyspnea & dyspnea & dyspnea & \\
\hline DVT & + & - & - & - & - & + \\
\hline Lupus AC & + & - & - & - & - & $+/-$ \\
\hline Anticardio AB & - & - & - & - & - & + \\
\hline D-dimer (ng/ml) & $647(\mathrm{nl}<220)$ & 8770 & 1600 & $>10,000$ & $\mathrm{n} / \mathrm{a}$ & $>10,000$ \\
\hline Albumin (g/dl) & $4.7(\mathrm{nl} 3.7-5.5)$ & 2.0 & 0.7 & 1.7 & 4.1 & 3.6 \\
\hline AT III (\%) & 105 (nl 77-132) & 278 & $7 / 2$ & 154 & $\mathrm{n} / \mathrm{a}$ & 114 \\
\hline Fibrinogen (ng/dl) & 718 (nl 180-394) & 234 & $\mathrm{n} / \mathrm{a}$ & 298 & $\mathrm{n} / \mathrm{a}$ & 421 \\
\hline
\end{tabular}

[PAH pulmonary arterial hypertension, CVA: cerebral vascular accident, AT III: anti-thrombin III, N/A: not available] All patients were treated with heparin and improved. No patient had any other genetic risk factors predisposing to thrombophilia.

Emory University School of Medicine, Atlanta, GA, USA

Full list of author information is available at the end of the article

(c) 2012 Vogler et al; licensee BioMed Central Ltd. This is an Open Access article distributed under the terms of the Creative Commons 
associated findings include nephrotic syndrome, elevated D-dimers and elevated fibrinogen levels. Recognition of PTE in pediatric patients with rheumatic diseases and prompt anti-coagulation therapy is important and potentially life-saving.

\section{Disclosure}

Larry B. Vogler: None; Sheila Angeles-Han: None; Sampath Prahalad: None; Egla C. Rabinovich: None.

\section{Author details}

'Duke University Medical Center, Durham, NC, USA. ${ }^{2}$ Emory Children's Center, Atlanta, GA, USA. ${ }^{3}$ Emory University, Atlanta, GA, USA. ${ }^{4}$ Emory University School of Medicine, Atlanta, GA, USA.

Published: 13 July 2012

doi:10.1186/1546-0096-10-S1-A73

Cite this article as: Vogler et al:: Pulmonary thromboembolism in

children with rheumatic diseases. Pediatric Rheumatology 2012 10(Suppl 1):A73.
Submit your next manuscript to BioMed Central and take full advantage of:

- Convenient online submission

- Thorough peer review

- No space constraints or color figure charges

- Immediate publication on acceptance

- Inclusion in PubMed, CAS, Scopus and Google Scholar

- Research which is freely available for redistribution

Submit your manuscript at www.biomedcentral.com/submit 